\title{
Computation of Milestones for Decision Support During System Restoration
}

\author{
Yunhe Hou, Member, IEEE, Chen-Ching Liu, Fellow, IEEE, Kai Sun, Member, IEEE, \\ Pei Zhang, Senior Member, IEEE, Shanshan Liu, Member, IEEE, and Dean Mizumura, Member, IEEE
}

\begin{abstract}
System restoration involves status assessment, optimization of generation capability, and load pickup. The optimization problem needs to take complex constraints into consideration, and therefore, it is not practical to formulate the problem as one single optimization problem. The other critical consideration for the development of decision support tools is its generality, i.e., the tools should be portable from a system to another with minimal customization. This paper reports a practical methodology for construction of system restoration strategies. The strategy adopted by each power system differs, depending on system characteristics and policies. A new method based on the concept of "generic restoration milestones (GRMs)" is proposed. A specific restoration strategy can be synthesized by a combination of GRMs based on the actual system conditions. The decision support tool is expected to reduce the restoration time, thereby improving system reliability. The proposed decision support tool has been validated with cases based on a simplified Western Electricity Coordinating Council (WECC) 200-Bus system and Hawaiian Electric Company system.
\end{abstract}

Index Terms-Generic restoration milestones, optimization, power system restoration.

\section{INTRODUCTION}

$\mathbf{P}$ OWER system restoration following a complete or partial outage is a highly complex decision and control problem. System restoration is a multistage process associated with different objectives and constraints. In this process, dispatchers in the control center work with field crews to re-establish the generation and transmission systems and then to pick up load and restore service [1], [2]. Statistical results show that the impact of a blackout increases with the duration of its restoration [3], [4] and, furthermore, as a result of cascading events, even a strong system can encounter vulnerable conditions that evolve into a catastrophic outage [5], [6]. To increase the robustness of a power system, a wide area protection and control system, such as the Strategic Power Infrastructure Defense system [7], is an

Manuscript received March 06, 2010; revised July 23, 2010; accepted September 10, 2010. Date of publication December 10, 2010; date of current version July 22, 2011. This work was supported by the Electric Power Research Institute (EPRI) and the Research Grant Council, Hong Kong SAR, under Grant 7124/10E. Paper no. TPWRS-00173-2010.

Y. Hou is with the Department of Electrical and Electronic Engineering, The University of Hong Kong, Hong Kong, China (e-mail: yhhou@eee.hku.hk).

C.-C. Liu is with the School of Electrical, Electronic, and Mechanical Engineering, University College Dublin, Dublin, Ireland (e-mail: liu@ucd.ie).

K. Sun, P. Zhang, and S. Liu are with Electric Power Research Institute, Palo Alto, CA 94304 USA (e-mail: ksun@epri.com; pzhang@epri.com; sliu@epri. com).

D. Mizumura is with the Hawaiian Electric Company, Inc., Honolulu, HI 96840 USA (e-mail: dean.mizumura@heco.com).

Digital Object Identifier 10.1109/TPWRS.2010.2089540 important tool. Several essential components, such as real-time sensing and communication, failure analysis, vulnerability assessment, and self-healing control, are involved in these systems. Power system restoration is an important component of the defense in-depth strategy. System reliability depends on the efficiency of system restoration. Much attention has been paid to R\&D in decision support tools for system restoration [1], [2], [8].

Few, if any, system-wide decision support tools are available to dispatchers and restoration planners at present. Restoration plans are developed with basic simulation tools for power flow, dynamics [3], [9], [10], and electromagnetic transients [11], [12]. These plans developed offline are used as guidelines for dispatchers in an online environment [13], [14]. Dispatchers need to adapt to actual outage scenarios and available resources and be able to develop the strategy based on their experience.

It is important to develop a decision support tool to assist power grids in system restoration planning and, ultimately, in an online restoration environment. Generally, the restoration strategies are tightly related with the characteristics of the systems [15]. As a result, restoration strategies for different systems differ significantly. Straightforward transplant of a restoration strategy from a system to another requires a significant level of customization. However, if one focuses on the tasks of power system restoration, different restoration strategies share some common characteristics.

The purpose of this paper is to discuss online decision support tools for system restoration. A new concept, called generic restoration milestones (GRMs) [16], for large interconnected power grids has been developed. A set of flexible GRMs and the associated algorithms that are able to evaluate different restoration strategy options are used to reconstruct system restoration strategies. A specific restoration strategy can be established by a combination of GRMs based on system characteristics, energy sources, and constraints. A different combination leads to a specific strategy option.

\section{Generic Restoration Milestones}

\section{A. Concept of GRMs}

The methodology proposed in this paper is abstracted from practices in industry. Generally, a procedure for system restoration has three stages, i.e., Preparation, System Restoration, and Load Restoration [4], [15]. According to these general stages of restoration, system restoration strategies can be categorized into five types, i.e., Build-Upward, Build-Downward, Build-Inward, Build-Outward, and Build-Together. Descriptions of these strategies can be found in [15]. To establish a restoration plan, 


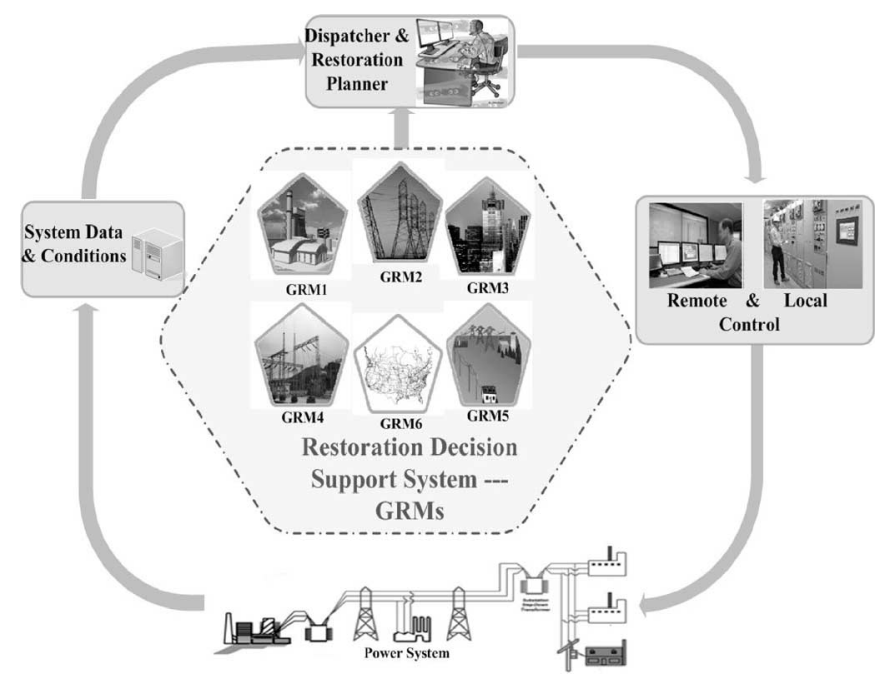

Fig. 1. Concept of GRMs.

the technical feasibility under both steady state and transient operating conditions need to be checked [8]. Technical constraints include: active power balance and frequency control, reactive power balance and overvoltage control, switching transient voltage [11], self-excitation, cold load pickup [17], system stability, protective systems, and load control [18].

The concept of GRMs [16] provides a toolbox to support system restoration planning and, ultimately, online system restoration. After analyzing system conditions and characteristics of an outage, system restoration planners or dispatchers select a series of Milestones from this toolbox to establish a restoration strategy. Following a power outage, dispatchers work with field crews to implement the restoration strategy established by a combination of GRMs. A comprehensive toolbox based on the concept of GRMs associated with highly-efficient algorithms provides an interactive system restoration decision support tool. This is illustrated in Fig. 1.

Generally, the following GRMs, GRM1-6, are needed:

- Form Black_Start_Non_Black_Start Building Block (GRM1)

- Establish Transmission Grid (GRM2)

- Form Electrical Island (GRM3)

- Synchronize Electrical Islands (GRM4)

- Serve Load in Area (GRM5)

- Connect with Neighboring System (GRM6)

A specific restoration strategy can be established by a combination of GRMs in the context of the system conditions. To implement GRMs, optimization algorithms incorporating system constraints are proposed in this paper.

\section{B. Algorithms for GRMs}

The objective of GRMs is to provide a comprehensive system restoration toolbox, and therefore, the related analysis tools may be included if needed.

GRM1: Form Black_Start_Non_Black_Start Building Block

Model 1: General Model of GRM1: The objective of GRM1 is to provide cranking power and restart available non-black start units as quickly as possible. Critical loads should be picked up as well. For implementation of GRM1, to maintain the stability of generating units and system voltage, some loads may be restored. Mathematically, the computational algorithm for GRM1 is described as follows.

Let $x_{i}$ denote the generating unit or critical load restarted at stage $S$, and $\boldsymbol{\theta}_{\mathbf{S}}$ be the set of all restarted generating units and critical loads at stage $S$. Let $f_{S}\left(x_{i}, \boldsymbol{\theta}_{\mathrm{S}}\right)$ be the shortest time to crank all generating units or critical loads after stage $S$. The recursive computation can be denoted as

$$
f_{S}\left(x_{i}, \boldsymbol{\theta}_{\mathbf{S}}\right)=\min _{x_{i} \in \boldsymbol{\theta}_{\mathbf{S}}}\left\{\Delta t_{x_{i}-x_{j}}+f_{S+1}\left(x_{j}, \boldsymbol{\theta}_{\mathbf{S}+\mathbf{1}}\right)\right\}
$$

where $x_{j}$ is the next generating unit to be restarted (so $x_{j}$ must be in $\boldsymbol{\theta}_{\mathbf{S}+\mathbf{1}}$ ). $\Delta t_{x_{i}-x_{j}}$ is the time to crank the generating unit (or critical load) $x_{j}$ from $\boldsymbol{\theta}_{\mathbf{S}+\mathbf{1}}$.

At each stage $S$, the technical constraints are represented as

$$
\begin{aligned}
& P F\left(\boldsymbol{\Omega}_{\mathbf{E}(\mathbf{S})}, \mathbf{P}_{\mathrm{G}(\mathbf{S})}, \mathbf{Q}_{\mathrm{G}(\mathbf{S})}, \mathbf{P}_{\mathbf{C L}(\mathbf{S})}\right.
\end{aligned}
$$

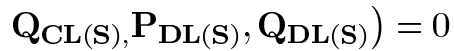

$$
\begin{aligned}
& \mathbf{P}_{\boldsymbol{\Pi}} \in \mathbf{F R}_{\mathbf{P}}(\boldsymbol{\Pi}), \mathbf{Q}_{\Pi} \in \mathbf{F R}_{\mathbf{Q}}(\boldsymbol{\Pi}) \\
& \mathbf{\Pi}=\mathbf{G}(\mathbf{S}), \mathbf{C L}(\mathbf{S}), \quad \text { and } \quad \mathbf{D L}(\mathbf{S}) \\
& \underline{V_{B}} \leq V_{B} \leq \overline{V_{B}}, \quad \forall B \in \Omega_{\mathbf{E}(\mathbf{S})} \\
& \underline{P_{L}} \leq P_{L} \leq \overline{P_{L}}, \quad \forall L \in \boldsymbol{\Omega}_{\mathbf{E}(\mathbf{S})}
\end{aligned}
$$

where energized block set $\boldsymbol{\Omega}_{\mathbf{E}(\mathbf{S})}$ includes all the energized buses and lines at stage $S, \mathbf{P}_{\mathrm{G}(\mathbf{S})}, \mathbf{Q}_{\mathrm{G}(\mathbf{S})}, \mathbf{P}_{\mathrm{CL}(\mathbf{S})}, \mathbf{Q}_{\mathrm{CL}(\mathbf{S})}, \mathbf{P}_{\mathrm{DL}(\mathbf{S})} \quad$ and $\mathrm{Q}_{\mathrm{DL}(\mathrm{S})}$ are vectors of real power of generating units, reactive power of generating units, real power of critical loads, reactive power of critical loads, real power of dispatchable loads, and reactive power of dispatchable loads, respectively. $P F(\cdot)$ is the power flow equations. $\mathbf{F R}_{\mathbf{P}}(\boldsymbol{\Pi})$ and $\mathbf{F R}_{\mathbf{Q}}(\boldsymbol{\Pi})$ denote feasible regions of real power and reactive power of the set $\Pi$. $\mathbf{G}(\mathbf{S}), \mathbf{C L}(\mathbf{S})$, and $\mathbf{D L}(\mathbf{S})$ are sets of generating units, critical loads, and dispatchable loads at stage $S$, respectively. $\Pi$ represents any one of these three sets. $\mathbf{P}_{\boldsymbol{\Pi}}$ and $\mathbf{Q}_{\boldsymbol{\Pi}}$ are real power and reactive power that belong to set $\Pi$, respectively. $V_{B}$ is the voltage at bus $B$, and $\underline{V_{B}}, \overline{V_{B}}$ are the corresponding lower and upper limits. $P_{L}$ is the real power flow on line $L$, and $P_{L}, \overline{P_{L}}$ are the corresponding lower and upper limits.

In this model, (2) represents the power flow equations at each stage of restoration; (3) shows that the real power and reactive power of each generating unit, critical load, and dispatchable load should stay within the feasible regions at each stage; (4) and (5) indicate that the voltage at each bus and power flow through each line should stay within limits. More constraints may be involved in this algorithm.

To solve this complex multistage optimization problem, a method with two interacting sub-problems is proposed. In these two sub-problems, an energized block of the system, i.e., $\Omega_{\mathbf{E}(\mathbf{S})}$, is determined by the primary problem, while the operating point for the block, i.e., $\mathbf{P}_{\mathbf{G}(\mathbf{S})}, \mathbf{Q}_{\mathrm{G}(\mathrm{S})}, \mathbf{P}_{\mathrm{CL}(\mathrm{S})}, \mathbf{Q}_{\mathrm{CL}(\mathrm{S})}, \mathbf{P}_{\mathrm{DL}(\mathrm{S})}$,

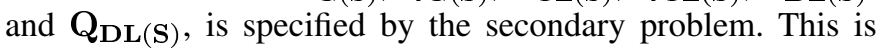
illustrated in Fig. 2. With this framework, practical constraints related to system operation are incorporated.

(i) Algorithms for the primary problem of GRM1 


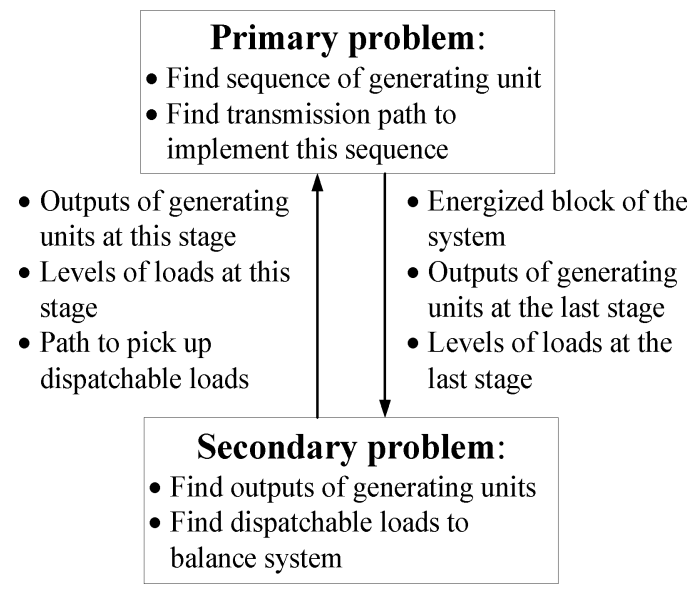

Fig. 2. Sub-problems in implementation of GRM1.

To find the sequence of generating units/critical loads and transmission path to implement this sequence, following two algorithms are involved.

\section{Algorithm 1: Finding a neighboring set of an energized block}

To avoid violations of system constraints, only generating units within a distance of an energized block are cranked during system restoration. An algorithm based on transformation of the connection matrix is proposed to find the neighboring set of an energized block. The following steps are used.

Step 1) Depth $=D, k=1$;

Step 2) Generate the connection matrix, i.e.,

$$
\begin{aligned}
& \mathbf{C M}(k) \\
& =\left[d_{i j}^{k}\right] \\
& = \begin{cases}1, & i=j \text { or } i \& j \text { are connected directly } \\
0, & i \& j \text { are not connected directly }\end{cases}
\end{aligned}
$$

where nonzero elements represent those buses that are involved in the block or connected with the block directly. Step 3) For the block $\Omega_{\mathrm{K}}$ at step $k$, establish the transformation matrix as

$$
\mathbf{T M}(k)=\left[t_{i j}^{k}\right]=\left\{\begin{array}{cc}
1, & i=j \text { or } \operatorname{line}_{i-j} \in \Omega_{\mathbf{K}} \\
0, & \text { else }
\end{array}\right.
$$

where elements of $\mathbf{T M}(k)$ indicate lines belong to $\Omega_{\mathbf{K}}$.

Step 4) $\mathbf{C M}(k+1) \leftarrow \mathbf{C M}(k) \cdot \mathbf{T M}(k)$;
According to the definition of $\mathbf{T M}(k), \mathbf{C M}(k) \cdot \mathbf{T M}(k)$ adds up all columns within the energized block. Therefore, each column represents the buses within this block with the same elements. Furthermore, according to (6), nonzero elements represent those buses that are involved in the block $\left(d_{i j}^{k}>1\right)$ or connected with the block directly $\left(d_{i j}^{k}=1\right)$. By detecting nonzero elements of $\mathbf{T M}(k+1)$, the buses within the block $\Omega_{\mathrm{K}}$ or connected with it directly will be found. Mathematically, it can be written as follows.

Step 5) $\forall j \in \boldsymbol{\Omega}_{\mathbf{K}}$, find set $\mathbf{I}^{\mathbf{K}+\mathbf{1}}=\left\{i \mid d_{i j}^{k} \neq 0\right.$ and $\left.i \notin \boldsymbol{\Omega}_{\mathbf{K}}\right\}$, where $\mathbf{I}^{\mathrm{K}+\mathbf{1}}$ is the set with buses connected with block $\boldsymbol{\Omega}_{\mathrm{K}}$ directly. Since all columns within the block are the same, the buses connected with the block directly can be detected from an arbitrary column.

Step 6) $\boldsymbol{\Omega}_{\mathbf{K}+1} \leftarrow \boldsymbol{\Omega}_{\mathbf{K}} \cup \mathbf{I}^{\mathbf{K}+\mathbf{1}}$

This step updates the buses involved in this block.

Step 7) $k \leftarrow k+1$. If $k \leq D$, go to step 3; else, output

$\Omega_{\mathrm{K}+1}$.

The idea of this algorithm is that nonzero elements of column $j$ indicate buses connected with bus $j$ directly. By generating TM $D$ times and evaluating CM - TM $D$ times, all buses connected with the block within $D$ lines will be found.

\section{Algorithm 2: Finding a transmission path to crank a generating unit}

To find transmission path to crank a generating unit, the charging current of each line is assigned as a weighting factor to avoid excessive charging currents (or steady-state over voltage). Usually, the charging current of a transformer is small. Therefore, a path with transformers may be selected by the search algorithm and, furthermore, a loop with more than one transformer might be selected to establish a cranking path. However, increasing the number of transformers on a path may increase the likelihood of ferroresonance. Hence, the weight of a branch with a transformer is set as a large number first. An extended algorithm based on Dijkstra's algorithm [19] is developed. Note that the basic Dijkstra's algorithm can only find the shortest path between two buses.

The following steps are used to find a path from the energized block set $\boldsymbol{\Omega}_{\mathbf{E}(\mathbf{S})}$ to an objective bus $B$.

Step 1) Establish the distance matrix, i.e., see (8) at the bottom of the page.

Step 2) $\forall i \in \boldsymbol{\Omega}_{\mathbf{E}}$, find the shortest path from $i$ to $B$ by Dijkstra's algorithm as $\left\{n_{k}, k=1,2, \ldots, m\right.$, and $\left.n_{1}=i\right\}$, where $n_{k}$ is a bus through the shortest path and the number of buses is $m$;

$$
\mathbf{D M}=\left[d_{i j}\right]=\left\{\begin{array}{lc}
0, & i \text { and } j \in \boldsymbol{\Omega}_{\mathbf{E}(\mathbf{S})} \\
\text { Charging current of line } \mathrm{i}-\mathrm{j}, & i \text { or } j \notin \boldsymbol{\Omega}_{\mathbf{E}(\mathbf{S})} \\
\text { a large number } \rho, \quad i-j \text { is a transformer and } i \text { or } j \notin \boldsymbol{\Omega}_{\mathbf{E}(\mathbf{S})}
\end{array}\right.
$$


Step 3) Find $n_{\lambda} \in \boldsymbol{\Omega}_{\mathbf{E}(\mathbf{S})}$ and $n_{\lambda+1} \notin \boldsymbol{\Omega}_{\mathbf{E}(\mathbf{S})}$, where $1 \leq \lambda<m$

In this step, $n_{\lambda+1}$ is the first bus outside the block and all buses within the path after $n_{\lambda+1}$ are outside the block.

Step 4) Output Path $=\left\{n_{k}\right\}, \quad k=\lambda+1, \lambda+2, \ldots, m$

The idea of this algorithm is to connect all buses within the block by zero length line first. Therefore, the shortest path from any bus within this block to the object bus is the shortest path from this block to that bus.

(ii) Algorithms for the secondary problem of GRM1

The purpose of the secondary problem is to meet constraints of power system operation for the energized block that is specified by the primary problem. The outputs of restarted generating units and dispatchable load levels are found in this sub-problem. An acceptable operating point, which satisfies all constraints, is determined based on the available ramping rates of generating units, load levels, and network structure. To accomplish this time critical GRM, the minimal time is used as the objective function. The major algorithms involved in this sub-problem are as follows.

\section{Algorithm 3: Finding an acceptable operating point of the system}

To minimize the duration, an optimal power flow (OPF) algorithm with minimal adjustment of each generating unit is established. Based on the ramping rate of a generating unit, the minimal adjustment corresponds to the shortest time to implement this objective:

$$
\min \sum_{P_{G i}^{(S)} \in \mathbf{P}_{\mathbf{G}(\mathbf{S})}}\left(P_{G i}^{(S)}-P_{G i}^{(S-1)}\right)^{2}
$$

Subject to

$$
\begin{aligned}
& \underline{P_{G i}^{(S)}} \leq P_{G i}^{(S)} \leq \overline{P_{G i}^{(S)}}, \quad \forall G i \in \mathbf{G}(\mathbf{S}) \\
& P F\left(\boldsymbol{\Omega}_{\mathrm{E}(\mathrm{S})}, \mathbf{P}_{\mathrm{G}(\mathrm{S})}, \mathbf{Q}_{\mathrm{G}(\mathrm{S})}, \mathbf{P}_{\mathrm{CL}(\mathbf{S})}\right. \\
& \left.\mathbf{Q}_{\mathrm{CL}(\mathbf{S})}, \mathbf{P}_{\mathbf{D L}(\mathbf{S})}, \mathbf{Q}_{\mathrm{DL}(\mathbf{S})}\right)=0 \\
& \mathbf{P}_{\boldsymbol{\Pi}} \in \mathbf{F R}_{\mathbf{P}}(\boldsymbol{\Pi}), \mathbf{Q}_{\Pi} \in \mathbf{F R}_{\mathbf{Q}}(\boldsymbol{\Pi}) \\
& \mathbf{\Pi}=\mathbf{G}(\mathbf{S}), \mathbf{C L}(\mathbf{S}) \text {, and } \mathbf{D L}(\mathbf{S}) \\
& \underline{V_{B}} \leq V_{B} \leq \overline{V_{B}}, \quad \forall B \in \Omega_{\mathbf{E}(\mathbf{S})} \\
& \underline{P_{L}} \leq P_{L} \leq \overline{P_{L}}, \quad \forall L \in \Omega_{\mathbf{E}(\mathbf{S})}
\end{aligned}
$$

where $P_{G i}^{(S)}$ and $P_{G i}^{(S-1)}$ are output of generating unit $G i$ at stage $S$ and $S-1$, respectively, $\overline{P_{G i}^{(S)}}$ and $P_{G i}^{(S)}$ are the upper and lower limits of the output of generating unit $G i$ at stage $S$. Other symbols are the same as the corresponding ones in (2)-(5).

In this problem, (10) is the limit of each generating unit, and it is determined by the upper limit and ramping rate of a generating unit; (11) represents power flow equations for the energized block at stage $S$; (12) describes physical constraints of each generating unit, critical load, and dispatchable loads; (13) and (14) are limits of voltage and power flow through each line

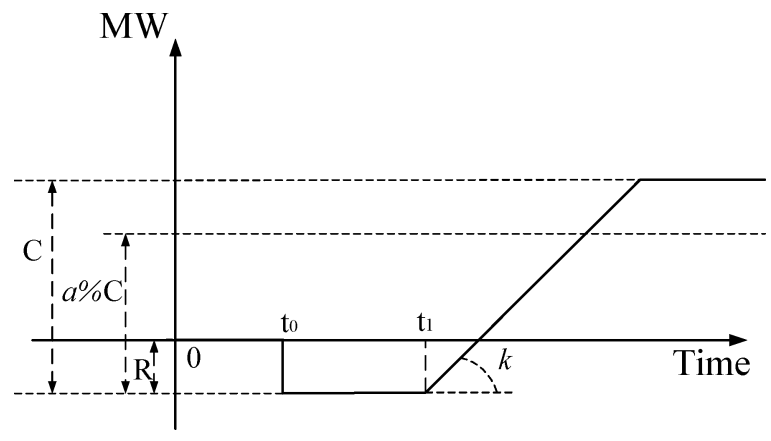

Fig. 3. Generic model of a generating unit.

at stage $S$. It should be noted that all constraints at stage $S-1$ are already met in the last step; only the constraints for stage $S$ are involved here.

The problem described by (9)-(14) is an OPF problem. To find a solution with a reasonable computing time, a vectorial interior point method is employed [20]. By the proposed algorithm, to balance the system at each stage, some dispatchable loads might be picked up.

Generally, the OPF algorithm is only implemented on a given network, i.e., only components that have been connected are involved in this computation. In this work, if the OPF defined by (9)-(14) is divergent, a proactive strategy is employed to find dispatchable loads to improve controllability of the system at each step during system restoration. The algorithm is described as follows.

\section{Algorithm 4: Finding dispatchable loads}

Step 1) Find the neighboring set of the energized block by Algorithm 1;

Step 2) Identify the dispatchable loads within the neighboring set;

Step 3) Find shortest path to crank the dispatchable loads by Algorithm 2

Step 4) Solve OPF problem defined by (9)-(14) with new dispatchable loads and associated transmission path. If the OPF problem can find a solution, output the solution; else, delete this state on the decision tree.

Model 2: General Model of Generating Units: The feasible regions of generating units not only depend on constraints of the system, but also the physical constraints. As a generic decision support system, characteristics of different generating units should be incorporated. A generic model of a generating unit is shown in Fig. 3. The parameters are described in Table I [15], [21].

Based on the physical constraints of generating units, a different combination of the parameters describes a different type of generating units [15], [21]. Furthermore, the critical load may be described as a generating unit with positive start-up power requirement and zero ramping rate. Therefore, for a generating unit, which is restarted at time $t_{0}$, the following equations hold:

$$
T_{3} \leq t_{0} \leq T_{2}
$$


TABLE I

PARAMETERS OF GENERATING UNITS

\begin{tabular}{ccccccc}
\hline \hline Cap. & $\begin{array}{c}\text { Start-up } \\
\text { requirement }\end{array}$ & Ramping & Min. & $\begin{array}{c}\text { Cranking } \\
\text { rate } \\
\text { output }\end{array}$ & $\begin{array}{c}\text { Critical } \\
\text { max. } \\
\text { paralleling }\end{array}$ & $\begin{array}{c}\text { Critical } \\
\text { Minterval }\end{array}$ \\
\hline$C$ & $R$ & $k$ & $\alpha \%$ & $T_{1}$ & $T_{2}$ & $T_{3}$ \\
interval
\end{tabular}

$$
T_{1}=t_{1}-t_{0}
$$

Generally, the generator output at time $t$ may be written as

$$
\begin{array}{r}
P(t)=\min \left\{k \cdot \max \left[t-\left(t_{0}+T_{1}\right), 0\right], C\right\} \\
-R \cdot U\left(t-t_{0}\right)
\end{array}
$$

where $U(\cdot)$ is a unit step function, which is defined as

$$
U(t)= \begin{cases}1, & t \geq 0 \\ 0, & t<0\end{cases}
$$

It can be validated that (17) represents the piecewise linear function described in Fig. 3.

\section{GRM2: Establish Transmission Grid}

To implement GRM2, the backbone transmission path is energized first, and sufficient load is restored to stabilize the system. During implementing of this GRM, system constraints are checked.

\section{Algorithm 5: Energizing transmission grid}

Step 1) Energize the backbone transmission path. A tree that connects all available buses is established. To maintain voltage and Var balance, the charging current is assigned as a weight to each line of the system. A subgraph, which connects all the buses together with a minimum sum of the weights of the lines, is found by a minimum spanning tree algorithm [19]. This formulation of the problem is as follows:

$$
\min _{\mathbf{\Omega}_{\mathbf{E}(\mathbf{S})}, \mathbf{\Omega}_{\mathbf{R}(\mathbf{S})} \subset \mathbf{E}(\mathbf{G})} \sum_{i=1}^{m} W_{i} C_{i}
$$

where $\boldsymbol{\Omega}_{\mathrm{E}(\mathrm{S})}$ is the set of all energized buses, $\boldsymbol{\Omega}_{\mathbf{R}(\mathrm{S})}$ is the set of remaining buses that will be connected at this step, $\mathbf{G}$ is the graph of this system, $\mathbf{E}(\mathbf{G})$ is the set of all buses in this graph, $W_{i}$ is the charging current of line $i, C_{i}$ is the state of line $i$, " 1 " means this line is connected and " 0 " means this line is not yet connected, and $m$ is the number of lines in this system. During implementation of this step, Algorithm 3 will be implemented when violations are detected.

Step 2) Establish an available transmission path.

Energization of each transmission path at this step results in closure of loops in the transmission grid. There is an abrupt change in generator power that occurs in nearby generators upon a loop closure. To reduce the impact, the difference of voltages between the nodes on both sides of the line should be sufficiently small. The algorithm based on the OPF problem is used to solve it [22].

The distinction between step 1 and step 2 is that during the second step, transmission loop closure is critical, and re-dispatch actions should be implemented to mitigate the impact, whereas at the first step, finding a strong backbone transmission path is the primary objective.

GRM3: Serve Load in Area

The objective function of this stage is to maximize the size of load pickup. The algorithm for knapsack problem is used at each stage. Constraints, such as frequency response, overvoltage, and overload, are considered. This problem is divided into a series of optimization problems at discrete time points. The objective is to maximize load pickup at each time point. The path for load pickup, load levels, and output of each generating unit are determined by this GRM. To solve this problem, the following algorithm is developed.

\section{Algorithm 6: Determining the level of load pickup}

Step 1) Determine candidate loads. Algorithms 1 is used to find the neighboring set of the energized block with a preset depth first, and loads within this set are detected as candidate loads. After implementation of this step, all candidate loads are detected. Let $\mathbf{R L}^{\mathbf{0}}(\mathbf{S})$ be a set consisted by all candidate loads.

Step 2) Determine locations for load pickup. The frequency response rates of prime movers for all generating units connected into the current system are detected first. By the algorithm proposed in [9], the upper limit of total load pickup is estimated. Finding loads that satisfy this constraint is a knapsack problem [19]; algorithms for knapsack problem are used to find the buses for loads pickup at this step. The loads found in this step form a subset of $\mathbf{R L}^{\mathbf{0}}(\mathbf{S})$. It is denoted as $\mathbf{R L}(\mathbf{S})$.

Step 3) Find paths for loads pickup. By Algorithms 2, the shortest path to pickup each load is found.

Step 4) Find load levels and outputs of generating units. The following OPF problem is established:

Subject to

$$
\max \sum_{P_{R L i}^{(S)} \in \mathbf{P}_{\mathbf{R L}(\mathbf{S})}} P_{R L i}^{(S)}
$$

$$
P_{R L i}^{(S-1)} \leq P_{R L i}^{(S)} \leq \overline{P_{R L i}^{(S)}}, \quad \forall R L i \in \mathbf{R L}(\mathbf{S})
$$

$$
\begin{gathered}
P F\left(\boldsymbol{\Omega}_{\mathrm{E}(\mathbf{S})}, \mathbf{P}_{\mathbf{R L}(\mathbf{S})}, \mathbf{Q}_{\mathbf{R L}(\mathbf{S})}, \mathbf{P}_{\mathrm{G}(\mathbf{S})}, \mathbf{Q}_{\mathrm{G}(\mathbf{S})}\right. \\
\left.\mathbf{P}_{\mathrm{CL}(\mathbf{S})}, \mathbf{Q}_{\mathbf{C L}(\mathbf{S})}, \mathbf{P}_{\mathbf{D L}(\mathbf{S})}, \mathbf{Q}_{\mathrm{DL}(\mathbf{S})}\right)=0
\end{gathered}
$$

$$
\begin{aligned}
& \mathbf{P}_{\boldsymbol{\Pi}} \in \mathbf{F R}_{\mathbf{P}}(\mathbf{\Pi}), \mathbf{Q}_{\Pi} \in \mathbf{F R}_{\mathbf{Q}}(\boldsymbol{\Pi}) \\
& \mathbf{\Pi}=\mathbf{G}(\mathbf{S}), \mathbf{C L}(\mathbf{S}), \mathbf{D L}(\mathbf{S}), \text { and } \mathbf{R L}(\mathbf{S}) \\
& \underline{V_{B}} \leq V_{B} \leq \overline{V_{B}}, \quad \forall B \in \mathbf{\Omega}_{\mathbf{E}(\mathbf{S})} \\
& \underline{P_{L}} \leq P_{L} \leq \overline{P_{L}}, \forall L \in \mathbf{\Omega}_{\mathbf{E}(\mathbf{S})}
\end{aligned}
$$


where $P_{R L i}^{(S)}$ and $P_{R L i}^{(S-1)}$ are load level of load $i$ at stage $S$ and $S-1$, respectively. $\mathbf{P}_{\mathbf{R L}(\mathbf{S})}$ and $\mathbf{Q}_{\mathbf{R L}(\mathbf{S})}$ are sets of active and reactive loads at stage $S$, respectively. $\mathbf{R L}(\mathbf{S})$ is the set of loads found at step 2. $\overline{P_{\mathrm{RL}}^{(S)}}$ is the upper limit of total load pickup at stage $S$. Other symbols are the same as the corresponding ones in (2)-(5). In this problem, different load models may be described by (21). Usually, picked-up loads cannot be shed. Equations (22)-(25) summarize the physical constraints of the power system at stage $S$.

\section{GRM4: Synchronize Electrical Islands}

According to the North American Electric Reliability Corporation (NERC) Standard: Emergency Preparedness and Operations(EOP)-005-1-System Restoration Plans, the islands can be synchronized with the surrounding island(s) when the following conditions are met: voltage, frequency, and phase angle permit; the size of the area being reconnected and the capacity of the transmission lines affecting the reconnection and the number of synchronizing points across the system are considered; Reliability Coordinator(s) and adjacent areas are notified and Reliability Coordinator approval is given; and load is shed in neighboring areas, if required, to permit successful interconnected system restoration. To address the above issues, several steps are taken in GRM4.

\section{Algorithm 7: Finding paths to synchronize electrical islands}

Step 1) Determine candidate substations. These substations must be equipped with synchroscopes that are needed for synchronizing two areas. They must have reliable communication with system dispatchers who direct the tie-in.

Step 2) Find a path to connect two substations for synchronization by Algorithm 2 .

Step 3) Crank the path for synchronization by the stronger system (the system with better adjustment capability) until the last breaker. Algorithm 3 is implemented when violations are detected at each line's closure.

Step 4) Adjust voltage and phase angle on both sides of the breaker for synchronization, and adjust the frequency of each island. All parameters should stay within allowable regions. Algorithm 3 is implemented when violations are detected.

Step 5) Synchronize two islands by closing the breaker.

\section{GRM5: Form Electrical Island}

The objective of GRM5 is to establish a strong island. The available loads will be picked up and transmission lines within this island will be energized. The same algorithms implementing GRM1, GRM2, and GRM3 are used here.

GRM6: Connect with Neighboring System

The algorithm to accomplish GRM6 is similar to the algorithms developed in GRM4. The difference between GRM6 and GRM4 is that in GRM6, the voltage in the neighboring system cannot be adjusted.

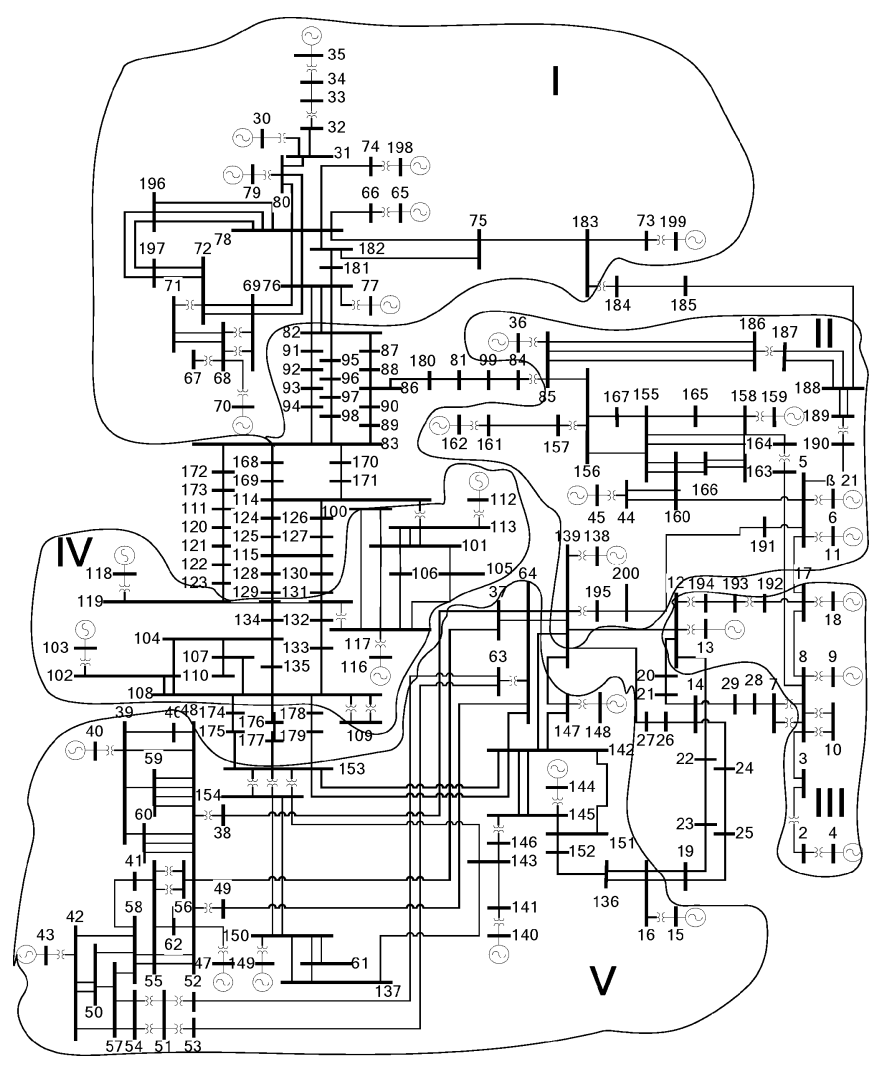

Fig. 4. Simplified WECC system.

TABLE II

Characteristics of CRITICAL LOADS OF THE 200-Bus SYSTEM

\begin{tabular}{ccc}
\hline \hline $\begin{array}{c}\text { Bus } \\
\text { No. }\end{array}$ & $\begin{array}{c}\text { Real Load } \\
\text { (p.u.) }\end{array}$ & $\begin{array}{c}\text { Reactive Power } \\
\text { (p.u.) }\end{array}$ \\
\hline 10 & 0.8 & 0.2 \\
155 & 2.75 & 0.5 \\
75 & 7.704 & -7.4 \\
\hline \hline
\end{tabular}

\section{Simulation RESUlts}

Two cases are used to demonstrate the capability of the proposed methodology.

\section{A. GRMs for a Simplified WECC System}

This case is based on a simplified 200-bus WECC system, as shown in Fig. 4. The characteristics of critical loads, dispatchable loads, and generating units are shown in Tables II-IV, respectively.

This system has 200 buses, 31 generating units, three critical loads, and five black start units. It is assumed that the system is operating in a normal state, where all the flows satisfy system operation constraints. The system has a total generation of 622 p.u. (real power) and 207.5 p.u. (reactive power). It has a total load of 503.8 p.u. (real power) and 151.3 p.u. (reactive power). Generating units on buses 13, 45, 116, 148, and 198 are black start units. Loads on buses 10,75 , and 155 are critical loads. It is assumed that the operating time to energize each line is $5 \mathrm{~min}$.

Based on the criteria of partitioning system into islands [23], this system is divided into five islands as shown in Fig. 4.

- Each island must have sufficient blackstart capability. 
TABLE III

CHARACTERISTICS of Dispatchable LOADS OF THE 200-Bus SyStem

\begin{tabular}{ccc}
\hline \hline $\begin{array}{c}\text { Bus } \\
\text { No. }\end{array}$ & $\begin{array}{c}\text { Min. / Max. Real } \\
\text { Load (p.u.) }\end{array}$ & $\begin{array}{c}\text { Min. / Max. Reactive } \\
\text { Power (p.u.) }\end{array}$ \\
\hline 34 & $0 / 25.6$ & $-2 / 6.2$ \\
31 & $0 / 30.4$ & $-4 / 8$ \\
78 & $0 / 45$ & $-20 / 40$ \\
183 & $0 / 10$ & $-10 / 10$ \\
188 & $0 / 2$ & $0 / 1$ \\
157 & $0 / 1$ & $-1 / 0.5$ \\
200 & $0 / 1$ & $-1 / 1.32$ \\
139 & $0 / 5.5$ & $-3 / 3$ \\
158 & $0 / 10$ & $-10 / 10$ \\
17 & $0 / 2$ & $-1 / 1$ \\
119 & $0 / 10$ & $-4 / 4$ \\
145 & $0 / 20$ & $-4 / 4$ \\
143 & $0 / 3$ & $-1 / 1$ \\
50 & $0 / 2$ & $-0.5 / 0.5$ \\
150 & $0 / 20$ & $-4 / 4$ \\
136 & $0 / 8$ & $-3 / 3$ \\
66 & $0 / 2.4$ & $-1.5 / 1.5$ \\
\hline \hline
\end{tabular}

TABLE IV

CHARACTERISTICS OF GENERATING UNITS OF THE 200-Bus System

\begin{tabular}{|c|c|c|c|c|c|c|c|c|}
\hline ID & Type & $\begin{array}{l}\text { Cap. } \\
\text { (p.u.) }\end{array}$ & $\begin{array}{l}\text { Ramp. Rate } \\
\text { (p.u. /hr) }\end{array}$ & $\begin{array}{c}\text { Crank } \\
\text { to } \\
\text { Paral. } \\
\text { (min.). }\end{array}$ & $\begin{array}{c}\text { Crit. } \\
\text { Min. } \\
\text { Int. } \\
\text { (min.) }\end{array}$ & $\begin{array}{c}\text { Crit } \\
\text { Max. } \\
\text { Int. } \\
\text { (min.) }\end{array}$ & $\begin{array}{c}\text { Start- } \\
\text { up } \\
\text { Req. } \\
\text { (p.u.) }\end{array}$ & $\begin{array}{l}\text { Min. } \\
\text { Output } \\
(\%)\end{array}$ \\
\hline 35 & NBS & 30 & 12 & 30 & N/A & inf & 2 & 50 \\
\hline 30 & NBS & 30 & 12 & 15 & N/A & inf & 1.5 & 30 \\
\hline 79 & NBS & 60 & 18 & 30 & N/A & inf & 3 & 50 \\
\hline 198 & BS & 15 & 6 & 0 & N/A & inf & 0 & 0 \\
\hline 65 & NBS & 20 & 18 & 15 & N/A & inf & 1.8 & 70 \\
\hline 199 & NBS & 15 & 6 & 0 & N/A & inf & 1.2 & 60 \\
\hline 77 & NBS & 45 & 15 & 30 & N/A & inf & 2.8 & 60 \\
\hline 70 & NBS & 30 & 15 & 0 & N/A & inf & 1.8 & 60 \\
\hline 36 & NBS & 36 & 18 & 30 & N/A & inf & 2 & 70 \\
\hline 162 & NBS & 8 & 10 & 0 & N/A & inf & 0.8 & 30 \\
\hline 159 & NBS & 8 & 10 & 0 & N/A & inf & 0.8 & 50 \\
\hline 45 & BS & 15 & 6 & 0 & N/A & inf & 0 & 0 \\
\hline 138 & NBS & 10 & 10 & 30 & N/A & $\inf$ & 1 & 20 \\
\hline 6 & NBS & 10 & 10 & 15 & N/A & inf & 1.2 & 30 \\
\hline 11 & NBS & 15 & 6 & 15 & N/A & inf & 1 & 20 \\
\hline 18 & NBS & 8 & 6 & 15 & N/A & inf & 0.6 & 50 \\
\hline 13 & BS & 8 & 6 & 0 & N/A & inf & 0 & 0 \\
\hline 9 & NBS & 8 & 6 & 25 & N/A & inf & 0.3 & 60 \\
\hline 4 & NBS & 15 & 12 & 15 & N/A & $\inf$ & 1 & 20 \\
\hline 118 & NBS & 30 & 24 & 30 & N/A & inf & 1.5 & 50 \\
\hline 103 & NBS & 12 & 12 & 0 & N/A & inf & 0.8 & 40 \\
\hline 116 & BS & 10 & 12 & 0 & N/A & inf & 0 & 0 \\
\hline 112 & NBS & 10 & 9 & 15 & N/A & $\inf$ & 1 & 20 \\
\hline 40 & NBS & 10 & 9 & 15 & N/A & inf & 1 & 20 \\
\hline 43 & NBS & 10 & 9 & 15 & $\mathrm{~N} / \mathrm{A}$ & inf & 1 & 20 \\
\hline 47 & NBS & 12 & 12 & 0 & N/A & inf & 0.8 & 40 \\
\hline 149 & NBS & 30 & 24 & 0 & N/A & $\inf$ & 1.5 & 50 \\
\hline 140 & NBS & 30 & 24 & 0 & $\mathrm{~N} / \mathrm{A}$ & inf & 1.5 & 50 \\
\hline 144 & NBS & 60 & 30 & 0 & $\mathrm{~N} / \mathrm{A}$ & inf & 3.5 & 50 \\
\hline 148 & BS & 10 & 12 & 0 & N/A & inf & 0 & 0 \\
\hline 15 & NBS & 12 & 12 & 30 & N/A & $\inf$ & 0.8 & 40 \\
\hline
\end{tabular}

- Each island should have enough cranking path to crank non-blackstart units or pick up loads.

- Each island should have the ability to match generation and load to within prescribed frequency limits.

- Each island should have adequate voltage controls to maintain a suitable voltage profile.
TABLE V

SEQUENCE FOR IMPLEMENTATION OF GRM1 IN ISLAND I

\begin{tabular}{ccccc}
\hline \hline Step & $\begin{array}{c}\text { Restoration } \\
\text { Action }\end{array}$ & $\begin{array}{c}\text { Time } \\
(\mathbf{m i n} .)\end{array}$ & Path & $\begin{array}{c}\text { Dispatchable } \\
\text { Loads } \\
\text { Picking up } \\
\text { (Bus No.) }\end{array}$ \\
\hline 1 & Restart BS on 198 & 0 & ----- & ---- \\
2 & Crank NBS on 77 & 31 & $198--74--78--182--$ & 78 \\
3 & Crank NBS on 30 & 47 & 181--76--77 & 31,78 \\
4 & Crank NBS on 65 & 72 & $78--66--65$ & $31,66,78$ \\
5 & Crank NBS on 70 & 98 & $76--69--68--70$ & $31,66,78$ \\
6 & Crank NBS on 79 & 114 & $80--79$ & $31,66,78$ \\
7 & Crank NBS on 35 & 145 & $31--32--33--34--35$ & $31,34,66,78,183$ \\
& Pick up critical & 156 & $182--75--183$ & \\
8 & load on 75 & & ----- & $31,34,66,78,183$ \\
9 & Crank NBS on 199 & 177 & $183--73--199$ & $31,34,66,78,183$ \\
\hline \hline
\end{tabular}

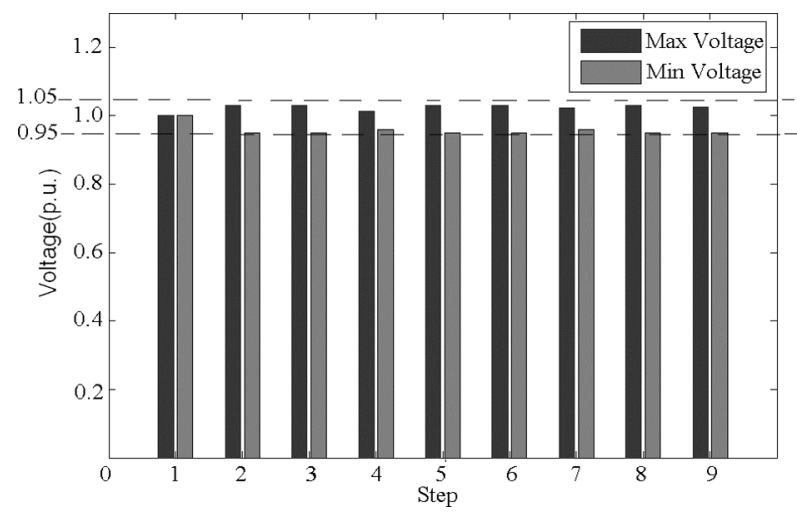

Fig. 5. Voltage profiles during implantation GRM1 for Island I.

- All tie points for subsystems must be capable of synchronization with adjacent subsystems.

- All islands should share information with other islands.

The restoration of this system involves five GRMs.

- Form Black_Start_Non_Black_Start Building Block

- Establish Transmission Grid

- Form Electrical Island

- Synchronize Electrical Islands

- Serve Load in Area

Use Island I as an example. The objective of GRM 1, i.e., Form Black_Start_Non_Black_Start Building Block, is to restart all generating units as quickly as possible. During the optimization process, the time to restart all generating units is minimized, and all constraints are satisfied. The sequence of actions for GRM1is shown in Table V.

By this sequence, all generating units in Islands I are restarted. Bus voltages together with the maximal and minimal voltages at each step are shown in Fig. 5. The power outputs are lower than the maximal output. No overvoltage is detected during the implementation of GRM1.

The objective of GRM1 is to minimize the duration of all generating units' restoration. For other sub-optimal sequence, i.e., 198-65-30-79-199-75-77-70-35, the outputs of sub-optimal and optimal sequence of Island I are shown in Fig. 6. By the optimal sequence, a shorter duration of restoration and a higher 


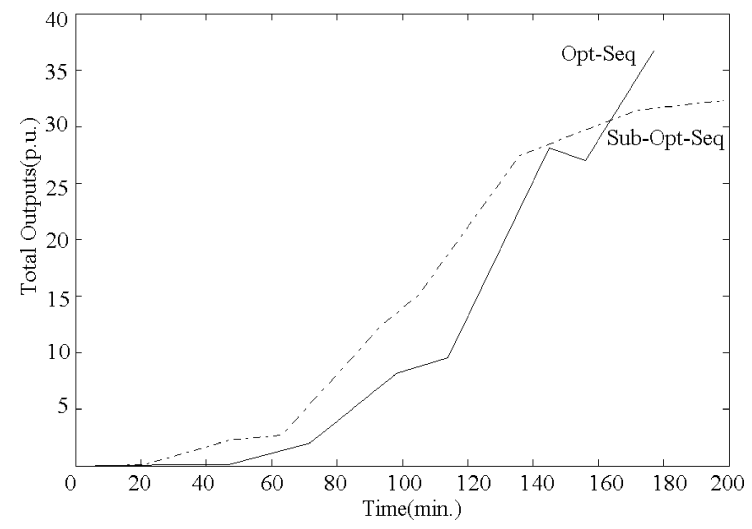

Fig. 6. Real power outputs of optimal and sub-optimal sequences.

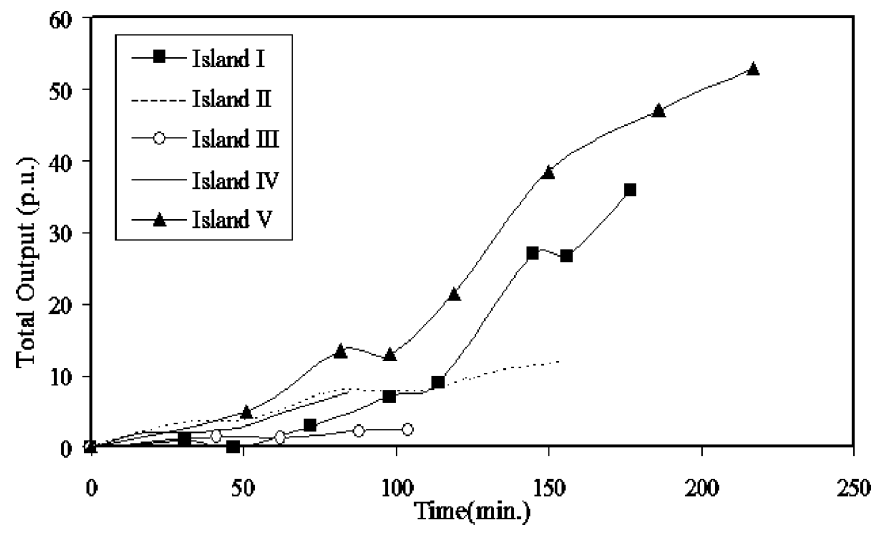

Fig. 7. Real power outputs of each island during implementation of GRM1.

output of all generating units are achieved. Due to the space limitation, only details of the restoration for Island I are provided here.

GRM1 is implemented on other islands. The outputs of optimal sequences of each island of the 200-bus system are given in Fig. 7.

After all islands are established, the islands can be synchronized, i.e., to implement GRM3. For instance, to synchronize Island I and Island II, the path for synchronization is found first. This path is: 76-82-87-88-86-180-81-99-84-85. The stronger system, i.e., Island I, will energize the path: 76-82-87-88-86-180-81-99-84. After the path is energized, the voltages of two buses for synchronization are: $\mathrm{V}_{84}=0.9513$ p.u. and $\mathrm{V}_{85}=0.9516$ p.u. The voltages of the island after synchronization are shown in Fig. 8.

It is shown that all voltages during synchronization are within the acceptable region.

The sequence and total output of the entire system for implementation of synchronization are given in Fig. 9.

Based on the proposed method, the entire system is restarted by a sequence of GRMs.

\section{B. GRMs for HECO's System}

A case study of the Hawaiian Electric Company (HECO) power system has been performed to test the proposed decision support tool. Restoration of the HECO transmission system in island Oahu is studied. Island Oahu experienced a blackout

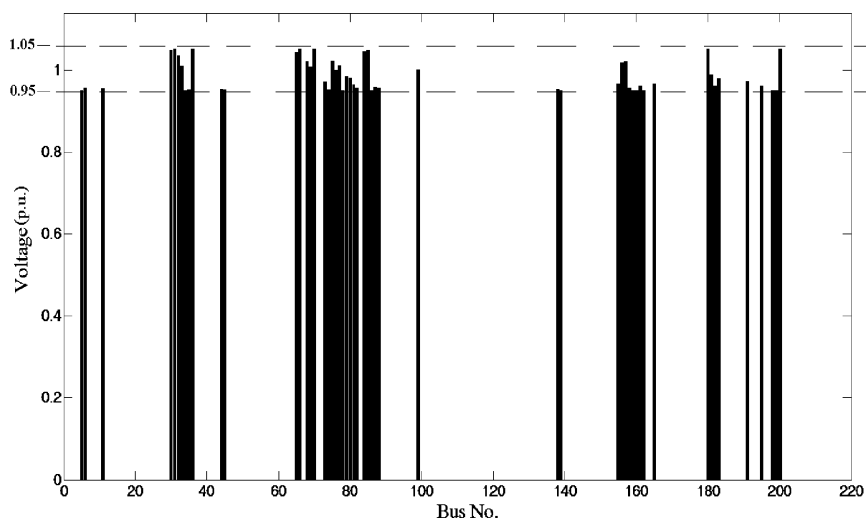

Fig. 8. Voltage profiles after synchronization of Island I and Island II.

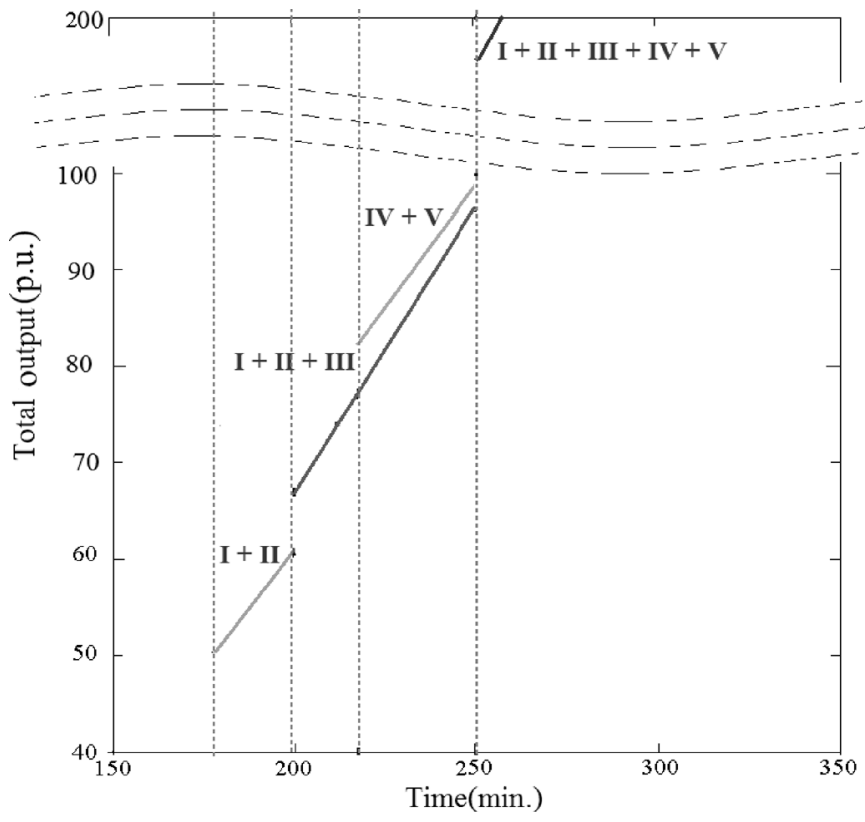

Fig. 9. Real power output of the entire 200-bus system during restoration.

due to the tremors of an earthquake on October 15, 2006. As shown in Fig. 10, its backbone transmission network with main power plants and substations (indicated, respectively, by circles and squares), between which branches denote one or multiple transmission lines. The system model considered in this case study has one blackstart unit at power plant $\mathrm{K} 1$ and a number of critical loads, e.g., airports and hospitals, connected to substations/plants K1, E1, W1, W2, K3, H2, M1, A2, P1, S1, I1, and A3 (indicated in gray color). The following three GRMs meet the requirement of HECO's practices in power system restoration:

- GRM1: Form Black_Start_Non_Black_Start Building Block

- GRM2: Establish Transmission Grid

- GRM5: Serve Load in Area

A benchmark restoration strategy is provided by HECO as described in Table VI. In the strategy, the transmission network is restored in the sequence of three target sub-systems indicated, respectively, by thick, thin, and dashed lines in Fig. 10. The 


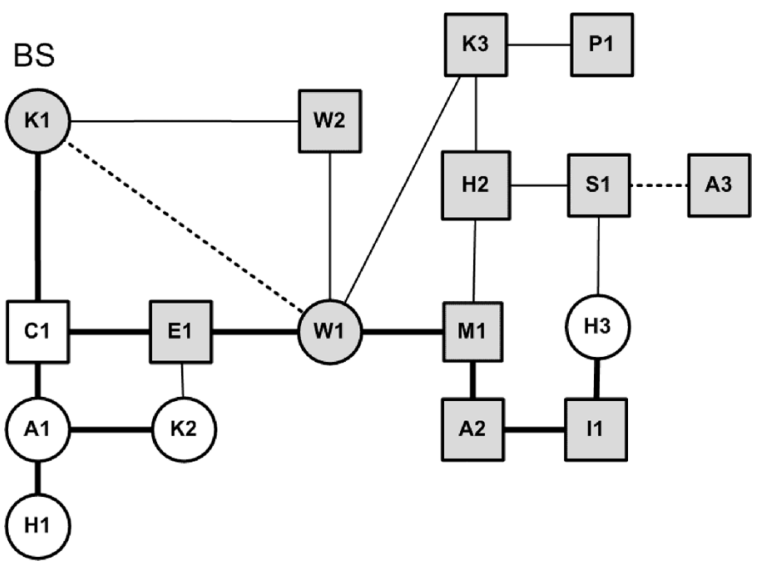

Fig. 10. Transmission network on island of Oahu.

TABLE VI

BENCHMARK AND GRM-BASED STRATEGIES FOR OAHU ISLAND SYSTEM

\begin{tabular}{|c|c|}
\hline Strategies & Descriptions of Restoration Path \\
\hline $\begin{array}{c}\text { Benchmark } \\
\text { strategy }\end{array}$ & $\begin{array}{l}\text { - Target Sub-system 1: } \\
\text { Starting from the BS at K1, energize plants/substations } \\
\text { in sequence: } \mathrm{C} 1, \mathrm{E} 1, \mathrm{~W} 1, \mathrm{M} 1, \mathrm{~A} 2, \mathrm{I} 1, \mathrm{~A} 1, \mathrm{~K} 2, \mathrm{H} 3 \text {, and } \\
\mathrm{H} 1 \text {; along that path, crank } 5 \mathrm{NBS} \text { units at } \mathrm{K} 1, \mathrm{~W} 1, \mathrm{~K} 2 \text {, } \\
\mathrm{H} 3 \text {, and ramp up their outputs and pick } \\
\text { critical/dispatchable loads to balance real-time } \\
\text { generation } \\
\text { - Target Sub-system 2: } \\
\text { Energize substations W2, H2, K3, P1 and S1; crank } \\
\text { another NBS unit at K2 and pick up } \\
\text { critical/dispatchable loads to balance real-time } \\
\text { generation } \\
\text { Target Sub-system 3: } \\
\text { Energize substation A3, crank the rest NBS units at } \\
\text { K2, W1, K1, A1, H3 and W1, and pick up the rest } \\
\text { critical loads }\end{array}$ \\
\hline $\begin{array}{c}\text { GRM- } \\
\text { Based } \\
\text { strategy }\end{array}$ & $\begin{array}{l}\text { - Target Sub-system 1: } \\
\text { Same as the above } \\
\text { - Target Sub-systems } \mathbf{2} \& \mathbf{3} \\
\text { Energize substations H2, K3, P1, W2, S1, A3 crank } \\
\text { the rest NBS units at A1, K1, W1, H3 and K2 and pick } \\
\text { up the rest critical loads }\end{array}$ \\
\hline
\end{tabular}

target sub-system 1 contains all power plants and several key substations, and the actions to restore that sub-system mainly focus on cranking non-blackstart units using the blackstart unit; the restoration actions for the two target sub-systems thereafter focus on picking up critical loads and establishing the transmission network.

This case study will compare the restoration strategy solved by the proposed decision support tool with that benchmark strategy. The study will allow certain relaxation on the sequence of target sub-systems in the benchmark strategy to explore alternative or more efficient restoration path. Since the target sub-system 1 is critical for reliable restoration, the proposed decision support tool is applied to only optimize the restoration path from accomplishing target sub-system 1 to achieve GRM1.

The simulation on the benchmark strategy shows that the total time cost for GRM1 is $438 \mathrm{~min}$. The strategy solved by the GRM-based decision support tool would take $364 \min (17 \%$

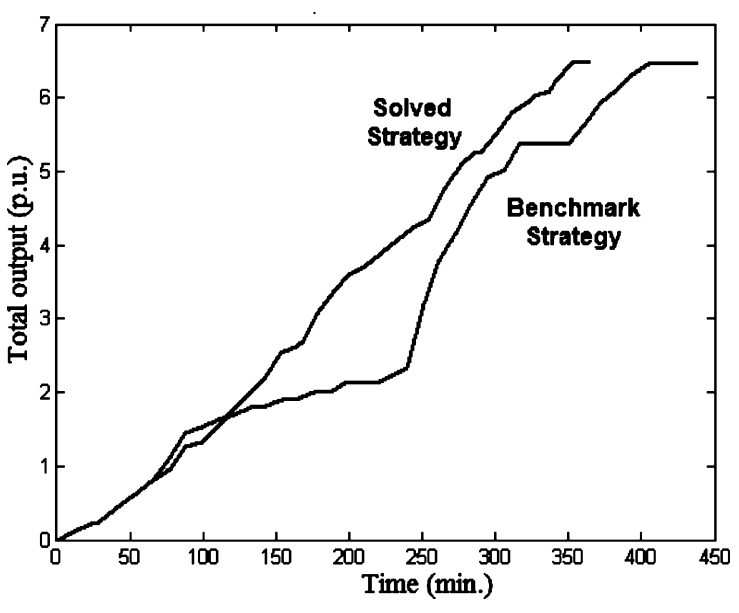

Fig. 11. Real power outputs for two strategies during implementation of GRM1.

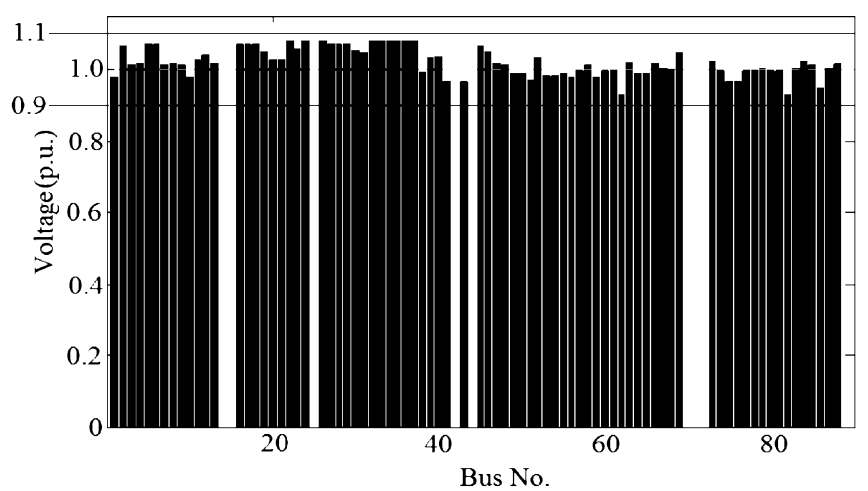

(a)

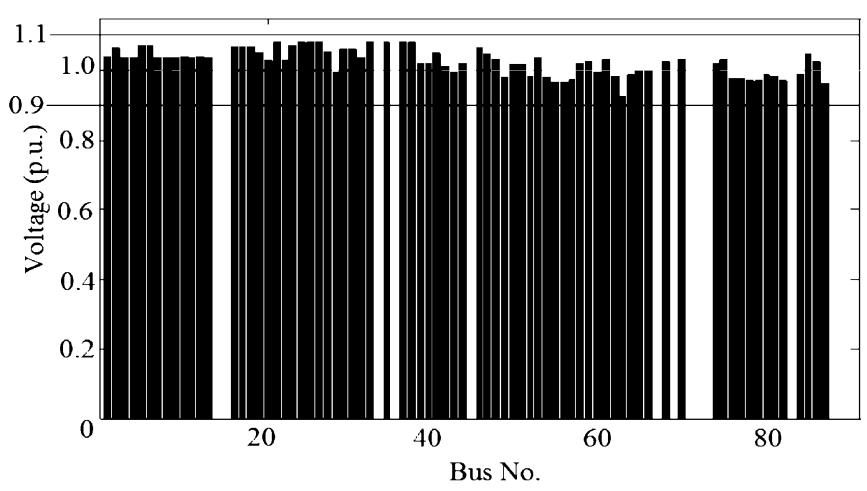

(b)

Fig. 12. Voltage profiles after GRM1 is accomplished. (a) Benchmark strategy. (b) GRM-based strategy.

less time) to achieve GRM1. The total generator outputs for two strategies are compared in Fig. 11. Their voltage profiles after GRM 1 is achieved are shown in Fig. 12. No voltage violation occurs.

It should be pointed out that the case study does not model all practical factors considered in HECO restoration plan, but the study results indicate that the decision support tool has the potential to assist restoration planners in developing alternative restoration path to energize power plants and substations and optimizing the sequence of cranking NBS units and picking up critical loads in the system. The strategies suggested by the 
tool may enable more prompt system restoration and could be starting points for further studies on improving existing strategies.

\section{CONCLUSION}

Restoration of a power system following a major outage is a complex, stressful, and time-consuming task. It is important to develop a decision support tool for evaluation of system restoration strategy options. The new concept of generic restoration milestones is abstracted from actual strategies of power system restoration. Based on system conditions, a specific restoration strategy can be established by a combination of GRMs. Based on the concept of GRMs, system restoration strategies need to be established under the supervision of dispatchers and system restoration planners. Computational algorithms have been developed to implement each GRM.

It is believed that the proposed GRM-based method is a practical approach to decision support for system restoration. The methodology represents a step toward modernization of power system restoration that is largely manual at present. However, practical implementation of the proposed method will depend on an assessment of power plants and network status in order to establish the specific GRMs and associated actions. Clearly, the assessment can be a major task during system restoration when uncertainty or lack of information is not uncommon. As system restoration progresses, it is inevitable that the system condition will change. When this situation arises, the proposed algorithms will allow GRMs to be modified for the new system condition.

\section{ACKNOWLEDGMENT}

The authors would like to thank the editor and reviewers for their valuable comments that have helped to improve the contents and readability of this paper.

\section{REFERENCES}

[1] M. M. Adibi, J. N. Borkoski, and R. J. Kafka, "Power system restoration-The second task force report," IEEE Trans. Power Syst., vol. 2, no. 4, pp. 927-932, Nov. 1987.

[2] M. Adibi, P. Clelland, L. Fink, H. Happ, R. Kafka, J. Raine, D. Scheurer, and F. Trefny, "Power system restoration-A task force report," IEEE Trans. Power Syst., vol. 2, no. 2, pp. 271-277, May 1987.

[3] M. M. Adibi and N. Martins, "Power system restoration dynamics issues," in Proc. IEEE Power Eng. Soc. General Meeting, Pittsburgh, PA, Jul. 20-24, 2008.

[4] M. M. Adibi and L. H. Fink, "Overcoming restoration challenges associated with major power system disturbances-Restoration from cascading failures," IEEE Power Energy Mag., vol. 4, no. 5, pp. 68-77, 2006.

[5] G. Andersson, P. Donalek, R. Farmer, N. Hatziargyriou, I. Kamwa, P. Kundur, N. Martins, J. Paserba, P. Pourbeik, J. Sanchez-Gasca, R. Schulz, A. Stankovic, C. Taylor, and V. Vittal, "Causes of the 2003 major grid blackouts in North America and Europe, and recommended means to improve system dynamic performance," IEEE Trans. Power Syst., vol. 20, no. 4, pp. 1922-1928, Nov. 2005.
[6] Y. V. Makarov, V. I. Reshetov, A. Stroev, and I. Voropai, "Blackout prevention in the United States, Europe, and Russia," Proc. IEEE, vol. 93, no. 11, pp. 1942-1955, Nov. 2005.

[7] H. Li, G. W. Rosenwald, J. Jung, and C.-C. Liu, "Strategic power infrastructure defense," Proc. IEEE, vol. 93, no. 5, pp. 918-933, May 2005.

[8] M. M. Adibi, Power System Restoration: Methodologies \& Implementation Strategies, ser. Power Engineering. New York: IEEE Press, 2000.

[9] M. M. Adibi, J. N. Borkoski, R. J. Kafka, and T. L. Volkmann, "Frequency response of prime movers during restoration," IEEE Trans. Power Syst., vol. 14, no. 2, pp. 751-756, May 1999.

[10] S. Spreng, H. Weber, and M. Hladky, "Investigation of the dynamic behaviour of hydro power plants for restoration scenarios," Int. J. Elect. Power, vol. 25, no. 8, pp. 615-621, 2003.

[11] M. M. Adibi, R. W. Alexander, and D. P. Milanicz, "Energizing high and extra-high voltage lines during restoration," IEEE Trans. Power Syst., vol. 14, no. 3, pp. 1121-1126, Aug. 1999.

[12] D. Lindenmeyer, H. W. Dommel, A. Moshref, and P. Kundur, "A framework for black start and power system restoration," in Proc. Can. Conf. Electrical and Computer Engineering, Halifax, NS, Canada, May 7-10, 2000

[13] C. Y. Teo and S. Wei, "Development of an interactive rule-based system for bulk power system restoration," IEEE Trans. Power Syst., vol. 15, no. 2, pp. 646-653, May 2000.

[14] M.-S. Tsai, "Development of an object-oriented service restoration expert system with load variations," IEEE Trans. Power Syst., vol. 23, no. 1, pp. 219-225, Feb. 2008.

[15] L. H. Fink, K.-L. Liou, and C.-C. Liu, "From generic restoration actions to specific restoration strategies," IEEE Trans. Power Syst., vol. 10, no. 2, pp. 745-752, May 1995.

[16] Y. Hou, C.-C. Liu, P. Zhang, and K. Sun, "Constructing power system restoration strategies," in Proc. Int. Conf. Electrical and Electronics Engineering, Bursa, Turkey, Nov. 2009.

[17] J. W. Feltes and C. Grande-Moran, "Black start studies for system restoration," in Proc. IEEE Power Eng. Soc. General Meeting, Pittsburgh, PA, Jul. 20-24, 2008.

[18] D. Lindenmeyer, H. W. Dommel, and M. M. Adibi, "Power system restoration-A bibliographical survey," Int. J. Elect. Power, vol. 23, no. 3, pp. 219-227, 2001.

[19] T. H. Cormen, C. E. Leiserson, R. L. Rivest, and C. Stein, Introduction to Algorithms, 2nd ed. Boston, MA: MIT Press and McGraw-Hill, 2001.

[20] Z. Qin, Y. Yang, and J. Wu, "Vectorial dynamic optimal power flow calculation including wind farms based on step-controlled primal-dual interior point method," in Proc. IEEE Int. Conf. Electrical Machines and Systems, Wuhan, China, Oct. 17-20, 2008.

[21] F. P. de Mello and J. C. Westcott, "Steam plant startup and control in system restoration," IEEE Trans. Power Syst., vol. 9, no. 1, pp. 93-101, Feb. 1994.

[22] N. Martins, E. J. de Oliveira, W. C. Moreira, J. L. R. Pereira, and R. M. Fontoura, "Redispatch to reduce rotor shaft impacts upon transmission loop closure," IEEE Trans. Power Syst., vol. 23, no. 2, pp. 592-600, May 2008.

[23] NERC's Standard-Emergency Preparedness and Operations. [Online]. Available: http://www.nerc.com.

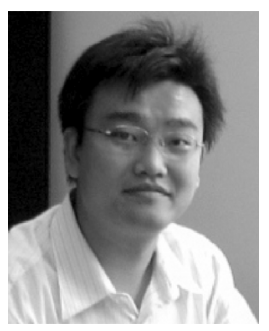

Yunhe Hou (M'06) received the B.E. and Ph.D. degrees from Huazhong University of Science and Technology, Wuhan, China, in 1999 and 2005, respectively.

He worked as a postdoctoral research fellow at Tsinghua University, Beijing, China, from 2005 to 2007. He was a visiting scholar at Iowa State University, Ames, and a research scientist at University College Dublin, Dublin, Ireland, from 2008 to 2009. $\mathrm{He}$ is currently with the University of Hong Kong as a Research Assistant Professor. He is also a visiting scientist with Laboratory for Information and Decision Systems, Massachusetts Institute of Technology (MIT), Cambridge, MA. 


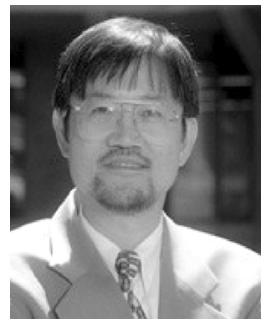

Chen-Ching Liu (S'80-M'83-SM'90-F'94) received the Ph.D. degree from the University of California, Berkeley.

He served as a Professor at the University of Washington, Seattle, from 1983-2005. During 2006-2008, he was Palmer Chair Professor at Iowa State University, Ames. Currently, he is a Professor of power systems at University College Dublin, Dublin, Ireland.

Prof. Liu received the IEEE PES Outstanding Power Engineering Educator Award in 2004. $\mathrm{He}$ served as Chair of the IEEE PES Technical Committee on Power System Analysis, Computing, and Economics during 2005-2006.

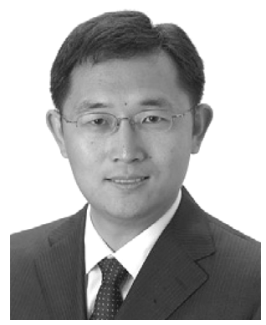

Kai Sun (M'06) received the B.S. degree in automation and the Ph.D. degree in control science and engineering from Tsinghua University, Beijing, China, in 1999 and 2004, respectively.

He was a Postdoctoral Research Associate at Arizona State University, Tempe, from 2005 to 2007. He is a Project Manager at EPRI, Palo Alto, CA.

Pei Zhang (SM'05) received the Ph.D. degree at Imperial College of Science, Technology, and Medicine, University of London, London, U.K., in 1999.

He worked with National Grid Company in the U.K. He is a Program Manager at EPRI, Palo Alto, CA. His research interests include power system stability, control, reliability, and security.

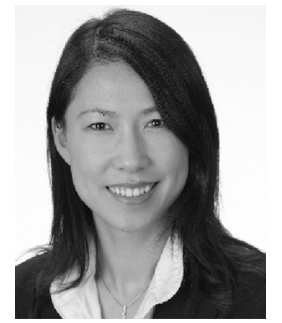

Shanshan Liu (M'09) received the Ph.D. degree from the University of Illinois, Urbana-Champaign.

She is currently a Senior Project Engineer Scientist in the Power System Analysis, Planning, and Operation group with Electric Power Research Institute (EPRI), Palo Alto, CA. Her current research activities focus on interactive system restoration, probabilistic risk assessment, and renewable integration.

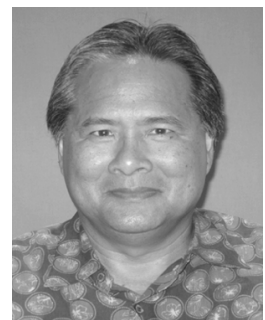

Dean Mizumura (M'75) received the B.S. degree in electrical engineering from Purdue University, West Lafayette, IN, in 1974 and the M.B.A. degree from the University of Hawaii, Honolulu, in 1990.

$\mathrm{He}$ served as the Operating Superintendent for the System Operation Department at Hawaiian Electric Company, Inc. from 1996 to 2007 and is currently the Acting Manager for the Asset Management Department. 